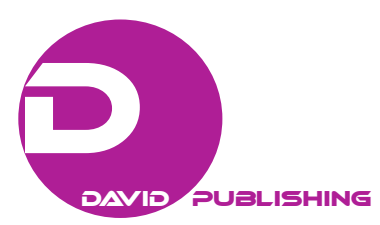

\title{
Task Assignment Problem of Robots in a Smart Warehouse
}

\section{Environment ${ }^{*}$}

\author{
Zhenping Li, Wenyu Li, Lulu Jiang \\ Beijing Wuzi University, Beijing, China
}

\begin{abstract}
The task assignment problem of robots in a smart warehouse environment (TARSWE) based on cargo-to-person is investigated. Firstly, the sites of warehouse robots and the order picking tasks are given and the task assignment problem for picking one order is formulated into a mathematical model to minimize the total operation cost. Then a heuristic algorithm is designed to solve the task assignment problem for picking multiple orders. Finally, simulations are done by using the orders data of online bookstore A. The results show that using the heuristic algorithm of this paper to assign robots, the cost was reduced by $2 \%$ and it can effectively avoid far route and unbalanced workload of robots. The feasibility and validity of the model and algorithm are verified. The model and algorithm in this paper provide a theoretical basis to solve the TARSWE.
\end{abstract}

Keywords: smart warehouse, robots, cargo-to-person, task assignment, mathematical model, heuristic algorithm

\section{Introduction}

The smart warehousing system based on "cargo-to-person" is a new type of distribution center warehousing management mode. In the smart warehousing system, items are stored in the movable shelves, and picking workers stand in front of stationary picking platform. Controlled by the computer system, the robot can lift up the assigned shelf and transport it to the closest picking platform. When picking and replenishing tasks are completed, the robot transports the shelf back to its original location. In the warehousing management system based on cargo-to-person, warehouse robots can complete complex works instead of human and the picking efficiency will be improved to some extent (Zou, 2013).

However, in the smart warehousing system, more complex tasks need to be completed by the robots. In order to improve the picking efficiency, the multi-robots cooperation is used in the general smart warehousing system. The intelligent warehousing system needs to coordinate multiple robots with multiple tasks at the same time, so the task assignment problem of warehouse robots is one of the key factors affecting the efficiency of warehousing system and needs to be studied deeply. In this paper, according to the orders which will be picked in a period of time, the task assignment problem of robots in a smart warehouse environment

\footnotetext{
* Project Supported: National Natural Science Foundation of China (11131009, 71540028, F012408), Funding Project for Academic Human Resources Development in Institutions of Higher Learning Under the Jurisdiction of Beijing Municipality (CIT\&TCD20130327), and major research project of Beijing Wuzi University.

Zhenping Li, professor, School of Information, Beijing Wuzi University, Beijing, China.

Wenyu Li, master, School of Information, Beijing Wuzi University, Beijing, China.

Lulu Jiang, master, School of Information, Beijing Wuzi University, Beijing, China.

Correspondence concerning this article should be addressed to Zhenping Li, No. 1 Fuhe Street, Tongzhou District, Beijing 101149, China.
} 
(TARSWE) based on cargo-to-person is investigated. Given the sites of warehouse robots and the tasks for picking one order, the task assignment problem of warehouse robots is formulated into a mathematical model to minimize the total operation cost. Then a heuristic algorithm is designed to solve the task assignment problem for picking multiple orders. Finally, simulations are done by using the simulated orders data of online bookstore A.

\section{Literature Review}

The "cargo-to-person"-based smart warehousing management mode has been proposed for several years and there are many theoretical issues remained to be studied (Wurman, Andrea, \& Mountz, 2008). So far, the researches about the TARSWE are still in a starting stage. The researches are mostly on the task allocation method for the warehouse robots. It mainly includes the method based on the market mechanism and the method based on swarm intelligence.

\section{Method Based on the Market Mechanism}

For solving the task assignment problem of a smart logistic center with robots, Gao (2006) studied the task allocation problem of robots by using the single item auction algorithm. Guo (2010) proposed a task assignment method based on the market auction idea. For heterogeneous robot completing different types of tasks, Kaleci and Parlaktuna (2012) proposed the task allocation method based on market mechanism. Trigui, Koubaa, and Cheikhrouhou (2014) thought that it can improve the work efficiency by switching tasks in the process of warehouse robots completing tasks and then proposed the extended distributed market oriented algorithm to solve the robot task allocation problem.

\section{Method Based on Swarm Intelligence}

Hazard, Wurman, and Andrea (2006) used "alphabet soup" to study the resource assignment problem of multi-vehicles system in the smart warehouse. $\mathrm{Li} \mathrm{(2012)} \mathrm{used} \mathrm{the} \mathrm{genetic} \mathrm{algorithm} \mathrm{and} \mathrm{ant} \mathrm{colony} \mathrm{algorithm} \mathrm{to}$ solve the TARSWE based on the traditional method of solving the multi traveling salesman problem. Liu (2010) proposed an orthogonal chaotic ant colony algorithm for dynamic multi robot task planning problem.

However, few studies are about the TARSWE.

\section{Research Methodology}

\section{Problem Description}

The task assignment problem of robots in the smart warehouse can be described as the follows: There are some isomorphic warehouse robots and several picking platforms in a smart warehouse with the picking mode of cargo-to-person; all the items are stored in the movable shelves according to their correlation $(\mathrm{Z}$. Li \& W. Li, 2014a), the movable shelves are neatly arranged in the warehouse, and each shelf is arranged in a determine site. There are several storages in each shelf and only one type of items stores in each storage. The coordinates of shelves, picking platforms, and all kinds of items stored in the warehouse are known. There are some aisles in the warehouse for the robots running smoothly. The fixed cost of calling each robot and the unit-distance cost of each warehouse robot walking loaded and unloaded are also known. During working time, each warehouse robot goes to an assigned shelf and transports it to the picking platform; after the workers picking items from the shelf, the robot transports it back to its original location; and the task is finished. Then, the robot can begin another task. Given a set of orders to be picked in a period of time $T$, the shelves to be moved for picking the 
orders are known. Suppose that the orders are picked one by one, the number of robots is larger than the maximum number of shelves that need to be transported for picking one order. In order to minimize the total operating costs, how to assign tasks for each warehouse robot is studied in this paper.

\section{Mathematical Model for Picking One Order}

Since the orders are picked one by one, firstly it formulates the task assignment problem of picking one order.

The symbols and variables are defined as follows:

$L=\left\{F_{1}, F_{2}, \ldots, F_{m}\right\}$ : set of available warehouse robots;

$B=\left\{b_{1}, b_{2}, \ldots, b_{k}\right\}:$ set of shelves in the warehouse, $b_{i}$ represents the $i$ th shelf;

$Q=\left\{t_{1}, t_{2}, \ldots, t_{n}\right\}$ : set of picking platform, $t_{j}$ represents the $j$ th picking platform;

$p$ : number of tasks for picking one order;

$S=\left\{s_{1}, s_{2}, \ldots, s_{p}\right\}:$ set of tasks for picking one order, $s_{i}$ represents the task of transport shelf $b_{i}$ to the picking platform;

$d_{i j}$ : walking distance of a robot walking from shelf $b_{i}$ to shelf $b_{j}, i=1,2, \ldots, p ; j=1,2, \ldots, p ;$

$u_{j}$ : walking distance of a robot transporting shelf $b_{j}$ to its corresponding picking platform, $j=1,2, \ldots, p$;

$r$ : fixed cost of each warehouse robot;

$c_{1}$ : unit-distance cost of a robot walking loaded with a shelf;

$c_{2}$ : unit-distance cost of a robot walking unloaded;

$x_{l h}=\left\{\begin{array}{l}1, \text { assign warehouse robot } F_{l} \text { to complete task } s_{h} ; \\ 0, \text { otherwise } l=1,2, \ldots, m ; h=1,2, \ldots, p .\end{array}\right.$

The total operation cost of warehouse robots completing all tasks is mainly composed of the related cost, the self-cost, and the fixed cost of calling each warehouse robot. Related cost refers to the cost that the robot needs to move unloaded from current position to position of a beginning task. Self-cost refers to the cost that the warehouse robot completes a task.

If the current position of a warehouse robot $F_{l}$ is in shelf $b_{i}$, assign $F_{l}$ to complete task $s_{j}$, then the cost of robot $F_{l}$ to finish task $s_{j}$ can be expressed as follows:

$$
\begin{aligned}
& c_{l j}=R C_{l}+S C_{l}+F C_{l} \\
& =d_{i j} c_{2}+2 u_{j} c_{1}+r \\
& \text { When } d_{i j} \geq A, c_{l j}=M \text {. }
\end{aligned}
$$

Among them, $R C_{l}$ is the related cost, $S C_{l}$ is the self-cost, and $F C_{l}$ is the fixed cost.

In order to establish a mathematical model, the $p$ tasks of an order are numbered from small to large according to the task subscript: $1,2, \ldots, p$. All the $c_{l j}$ form a vector matrix $C_{m p}$ of $m$ row and $p$ column, among them, the element $c_{l h}$ refers to the cost of warehouse robot $F_{l}$ completing the task $s_{h}$.

Based on the above analysis, after the robots completing all tasks of an order, they will be assigned to complete new tasks. In order to minimize the total operation cost, the new tasks assignment problem of warehouse robots can be formulated into the following integer linear programming model. 


$$
\begin{gathered}
\min z=\sum_{l=1}^{m} \sum_{h=1}^{p} c_{l h} x_{l h} \\
\text { s.t. }\left\{\begin{array}{l}
\sum_{l=1}^{m} x_{l h}=1, \quad h=1,2, \ldots, p \\
\sum_{h=1}^{p} x_{l h} \leq 1, \quad l=1,2, \ldots, \mathrm{m} \\
x_{l h}=0,1, \quad l=1,2, \ldots, m, h=1,2, \ldots, p
\end{array}\right.
\end{gathered}
$$

Objective function (1) minimizes the total operation cost; constraint (2) refers to that each task can be assigned to one warehouse robot; constraint (3) represents that every warehouse robot can complete no more than one task; and constraint (4) indicates that the variables are binary.

The mathematical model of one order picking task assignment problem is a 0-1 programming model, which can be solved in polynomial time, for example, the improved Hungarian algorithm can be used to solve the integer programming model and obtain the exact optimal solution (Wu \& Dong, 2008).

\section{Heuristic Algorithm for Picking Multiple Orders}

The set of orders to be picked in a period of time $T$ is given and the orders are usually divided into several batches: Each batch is picked at the same time, since the number of tasks for picking all orders is much larger than the number of warehouse robots. This paper assumes that every batch consists of one order, which means that the orders are picked one by one.

Based on this assumption, a heuristic algorithm for picking multiple orders is designed.

Suppose there are $e$ orders to be picked, which are listed in a set:

$$
O=\left\{O_{1}, O_{2}, \ldots, O_{e}\right\}
$$

The tasks for picking each order are known.

The heuristic algorithm for picking all orders can be described as follows:

Step 1: Select the first order from order list set $O$; according to the warehouse robot's position and the tasks for picking the first order, calculate the cost matrix $C_{l p}$. Then, the optimal task assignment solution for picking the first order can be obtained by solving the integer programming model in section 2.2.

Step 2: Update the positions of warehouse robots. The positions of robots that participate in picking tasks of the first order are updated as their corresponding task position. Positions of warehouse robots that do not participate in picking the first order remain unchanged. Delete the first order set.

Step 3: If the order set is empty, go to step 4; else go to step 1.

Step 4: Output the tasks assignment solution for picking all orders.

\section{Results Analysis}

The methods are tested by analyzing the orders data of online bookstore A.

\section{Description of Online Bookstore A}

There are 96 classes of books sold in online bookstore A stored in 24 movable shelves and each movable shelf has two layers. Each layer has two storage spaces and each storage space stores one class of books, namely, there are at most four classes of books stored in each shelf. At present, A bookstore uses the smart warehousing system based on cargo-to-person to manage picking and replenishment operation. There are three 
picking platforms in the warehouse. The warehouse has 10 warehouse robots walking along a specific landmark. In the current operation mode, the orders are picked one by one, which means that the next order would not be picked until the picked order is packaged, so each order requires a separate picking time.

As shown in Figure 1, Cartesian coordinates system is established. The warehouse is abstracted as a grid map and each box in the figure is abstracted into a position coordinates $(x, y)$. Square boxes represent shelves, the cycles with arrow represent warehouse robots, and the rectangular boxes represent picking platforms.

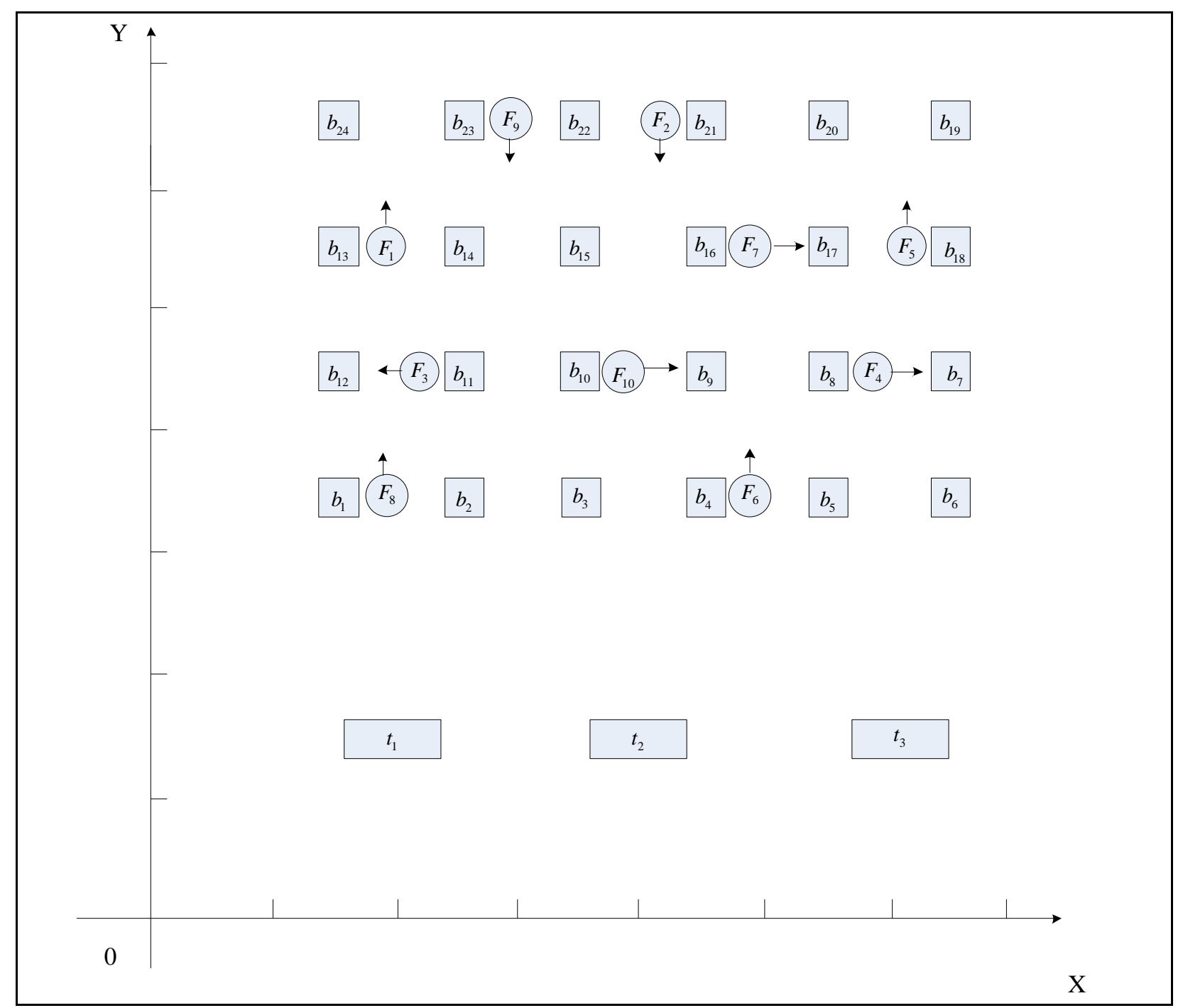

Figure 1. Warehouse layout simplified diagram of online bookstore A at 10 a.m. (coordinate unit: 2 m).

It simulated 10 orders to be picked between 10 a.m. and 12 a.m. The corresponding tasks sets of 10 orders are listed in Table 1. Coordinates of shelves and picking platforms are listed in Table 2. Thus, the walking distance among any pair of shelves and between the shelf and its corresponding picking platform can be calculated. The detailed information of 10 orders is known. The unit-distance cost of each warehouse robot walking loaded and unloaded are 3 dollars and 1.9 dollars and the fixed cost of calling each warehouse robot is 10 dollars. In order to avoid robot collision, assuming that $c_{i j}$ is infinity if $d_{i j} \geq 10$. According to the existing 
resource in the warehouse, in order to minimize the total operation cost, how are tasks assigned for each warehouse robot?

Table 1

The Corresponding Task Sets of 10 Orders

\begin{tabular}{ll}
\hline Order & Task sets of the order $(S)$ \\
\hline 1 & $\left\{s_{2}, s_{5}, s_{10}, s_{16}\right\}$ \\
2 & $\left\{s_{2}, s_{6}, s_{7}, s_{8}, s_{10}, s_{21}, s_{23}, s_{24}\right\}$ \\
3 & $\left\{s_{4}, s_{8}, s_{12}, s_{18}, s_{20}, s_{22}, s_{24}\right\}$ \\
4 & $\left\{s_{3}, s_{9}, s_{14}, s_{18}, s_{19}, s_{22}, s_{24}\right\}$ \\
5 & $\left\{s_{1}, s_{3}, s_{5}, s_{11}, s_{14}, s_{15}, s_{19}, s_{23}\right\}$ \\
6 & $\left\{s_{2}, s_{5}, s_{9}, s_{11}, s_{16}, s_{19}, s_{23}\right\}$ \\
7 & $\left\{s_{4}, s_{7}, s_{10}, s_{13}, s_{17}, s_{18}\right\}$ \\
8 & $\left\{s_{2}, s_{8}, s_{14}, s_{22}, s_{23}\right\}$ \\
9 & $\left\{s_{2}, s_{6}, s_{11}, s_{12}, s_{14}, s_{19}, s_{22}, s_{23}\right\}$ \\
10 & $\left\{s_{3}, s_{6}, s_{7}, s_{12}, s_{17}, s_{21}\right\}$ \\
\hline
\end{tabular}

Table 2

Coordinates of Each Shelf and Each Picking Platform

\begin{tabular}{lllll}
\hline Shelf & $b_{1}$ & $b_{2}$ & $b_{3}$ & $b_{4}$ \\
\hline Coordinate & $(3,7)$ & $(5,7)$ & $(7,7)$ & $(9,7)$ \\
Shelf & $b_{5}$ & $b_{6}$ & $b_{7}$ & $b_{8}$ \\
Coordinate & $(11,7)$ & $(13,7)$ & $(13,9)$ & $(11,9)$ \\
Shelf & $b_{9}$ & $b_{10}$ & $b_{11}$ & $b_{12}$ \\
Coordinate & $(9,9)$ & $(7,9)$ & $(5,9)$ & $(3,9)$ \\
Shelf & $b_{13}$ & $b_{14}$ & $b_{15}$ & $b_{16}$ \\
Coordinate & $(3,11)$ & $(5,11)$ & $(7,11)$ & $(9,11)$ \\
Shelf & $b_{17}$ & $b_{18}$ & $b_{19}$ & $b_{20}$ \\
Coordinate & $(11,11)$ & $(13,11)$ & $(13,13)$ & $(11,13)$ \\
Shelf & $b_{21}$ & $b_{22}$ & $b_{23}$ & $b_{24}$ \\
Coordinate & $(9,13)$ & $(7,13)$ & $(5,13)$ & $(3,13)$ \\
Picking platform & $t_{1}$ & $t_{2}$ & $t_{3}$ & \\
Coordinate & $(4,3)$ & $(8,3)$ & $(12,3)$ & \\
\hline
\end{tabular}

\section{Results Analysis of Online Bookstore A}

At first, according to the coordinates of shelves and picking platforms in Table 2, the walking distance among any two shelves and between the shelf and its corresponding picking platform are calculated. Then the algorithm in this paper is used to get the task assignment schemes of the warehouse robots.

For the first order, according to the coordinates of shelves and picking platforms, the vector matrix $C_{10,4}$ can be obtained ( $M$ denotes infinity). 


$\left[\begin{array}{lccc}51.4 & M & 63.4 & 75.4 \\ M & 55.2 & 63.4 & 67.8 \\ 43.8 & 55.2 & 55.8 & 75.4 \\ 55.2 & 43.8 & 59.6 & 71.6 \\ M & 51.4 & 67.2 & 71.6 \\ 47.6 & 43.8 & 59.6 & 71.6 \\ 55.2 & 51.4 & 59.6 & 64 \\ 43.8 & 55.2 & 63.4 & M \\ 51.4 & M & 63.4 & 75.4 \\ 47.6 & 51.4 & 52 & 71.6\end{array}\right]$

Then solving the integer programming model by using the LINGO program, the optimal task assignment schemes of order 1 can be obtained: $F_{3} \rightarrow b_{2} ; F_{4} \rightarrow b_{5} ; F_{10} \rightarrow b_{10} ; F_{7} \rightarrow b_{16}$, and the cost is $43.8+43.8+52+64$ $=203.6$ dollars. Where, $F_{l} \rightarrow b_{j}$ represents that warehouse robot $F_{l}$ is assigned to transport shelf $b_{j}$ to the corresponding picking platform.

After finishing all the tasks of order 1, the positions of warehouse robots are updated. Then using the same method, the optimal task assignment schemes of order 2 to order 10 can be obtained. The results are shown in Table 3.

According to the corresponding task assignment schemes and cost of robots for picking each order in Table 3, the total operation cost is 4,010.4 dollars, which the warehouse robots spend on completing all tasks within 10 a.m. and 12 a.m.

At present, the task assignment of warehouse robots of online bookstore A is made according to personnel experience, which might lead to the long route and unbalanced workload of different robots. The specific task assignment schemes within 10 a.m. and 12 a.m. are shown in Table 4.

Table 3

Corresponding Task Assignment Schemes and Cost of Warehouse Robots of Each Order by the Heuristic Algorithm

\begin{tabular}{lll}
\hline Order & Task assignment schemes & Cost (dollars) \\
\hline 1 & $F_{3} \rightarrow b_{2} ; F_{4} \rightarrow b_{5} ; F_{10} \rightarrow b_{10} ; F_{7} \rightarrow b_{16}$ & 203.6 \\
2 & $F_{3} \rightarrow b_{2} ; F_{6} \rightarrow b_{6} ; F_{5} \rightarrow b_{7} ; F_{4} \rightarrow b_{8} ; F_{10} \rightarrow b_{10} ; F_{2} \rightarrow b_{21} ; F_{9} \rightarrow b_{23} ; F_{1} \rightarrow b_{24}$ & 483 \\
3 & $F_{3} \rightarrow b_{4} ; F_{4} \rightarrow b_{8} ; F_{8} \rightarrow b_{12} ; F_{5} \rightarrow b_{18} ; F_{2} \rightarrow b_{20} ; F_{9} \rightarrow b_{22} ; F_{1} \rightarrow b_{24}$ & 458.8 \\
4 & $F_{3} \rightarrow b_{3} ; F_{7} \rightarrow b_{9} ; F_{8} \rightarrow b_{14} ; F_{5} \rightarrow b_{18} ; F_{2} \rightarrow b_{19} ; F_{9} \rightarrow b_{22} ; F_{1} \rightarrow b_{24}$ & 455 \\
5 & $F_{7} \rightarrow b_{1} ; F_{3} \rightarrow b_{3} ; F_{4} \rightarrow b_{5} ; F_{10} \rightarrow b_{11} ; F_{8} \rightarrow b_{14} ; F_{9} \rightarrow b_{15} ; F_{2} \rightarrow b_{19} ; F_{1} \rightarrow b_{23}$ & 482.4 \\
6 & $F_{7} \rightarrow b_{2} ; F_{4} \rightarrow b_{5} ; F_{3} \rightarrow b_{9} ; F_{10} \rightarrow b_{11} ; F_{9} \rightarrow b_{16} ; F_{2} \rightarrow b_{19} ; F_{1} \rightarrow b_{23}$ & 415.2 \\
7 & $F_{4} \rightarrow b_{4} ; F_{6} \rightarrow b_{7} ; F_{3} \rightarrow b_{10} ; F_{8} \rightarrow b_{13} ; F_{9} \rightarrow b_{17} ; F_{5} \rightarrow b_{18}$ & 355 \\
8 & $F_{7} \rightarrow b_{2} ; F_{6} \rightarrow b_{8} ; F_{8} \rightarrow b_{14} ; F_{3} \rightarrow b_{22} ; F_{1} \rightarrow b_{23}$ & 323.2 \\
9 & $F_{4} \rightarrow b_{2} ; F_{5} \rightarrow b_{6} ; F_{7} \rightarrow b_{11} ; F_{10} \rightarrow b_{12} ; F_{8} \rightarrow b_{14} ; F_{2} \rightarrow b_{19} ; F_{3} \rightarrow b_{22} ; F_{1} \rightarrow b_{23}$ & 498.8 \\
10 & $F_{4} \rightarrow b_{3} ; F_{5} \rightarrow b_{6} ; F_{6} \rightarrow b_{7} ; F_{10} \rightarrow b_{12} ; F_{9} \rightarrow b_{17} ; F_{3} \rightarrow b_{21}$ & 335.4 \\
\hline
\end{tabular}


Table 4

Corresponding Task Assignment Schemes and Cost of Warehouse Robots of Each Order in Practice

\begin{tabular}{lll}
\hline Order & Task assignment schemes & Cost (dollars) \\
\hline 1 & $F_{8} \rightarrow b_{2} ; F_{4} \rightarrow b_{5} ; F_{10} \rightarrow b_{10} ; F_{7} \rightarrow b_{16}$ & 203.6 \\
2 & $F_{8} \rightarrow b_{2} ; F_{4} \rightarrow b_{6} ; F_{4} \rightarrow b_{7} ; F_{6} \rightarrow b_{8} ; F_{10} \rightarrow b_{10} ; F_{2} \rightarrow b_{21} ; F_{9} \rightarrow b_{23} ; F_{1} \rightarrow b_{24}$ & 483 \\
3 & $F_{3} \rightarrow b_{4} ; F_{6} \rightarrow b_{8} ; F_{8} \rightarrow b_{12} ; F_{5} \rightarrow b_{18} ; F_{7} \rightarrow b_{20} ; F_{10} \rightarrow b_{22} ; F_{1} \rightarrow b_{24}$ & 466.4 \\
4 & $F_{8} \rightarrow b_{3} ; F_{3} \rightarrow b_{9} ; F_{9} \rightarrow b_{14} ; F_{5} \rightarrow b_{18} ; F_{7} \rightarrow b_{19} ; F_{10} \rightarrow b_{22} ; F_{1} \rightarrow b_{24}$ & 470.8 \\
5 & $F_{6} \rightarrow b_{1} ; F_{8} \rightarrow b_{3} ; F_{8} \rightarrow b_{5} ; F_{3} \rightarrow b_{11} ; F_{9} \rightarrow b_{14} ; F_{10} \rightarrow b_{15} ; F_{7} \rightarrow b_{19} ; F_{1} \rightarrow b_{23}$ & 493.8 \\
6 & $F_{6} \rightarrow b_{2} ; F_{8} \rightarrow b_{5} ; F_{4} \rightarrow b_{9} ; F_{3} \rightarrow b_{11} ; F_{10} \rightarrow b_{16} ; F_{7} \rightarrow b_{19} ; F_{1} \rightarrow b_{23}$ & 415.2 \\
7 & $F_{8} \rightarrow b_{4} ; F_{4} \rightarrow b_{7} ; F_{3} \rightarrow b_{10} ; F_{9} \rightarrow b_{13} ; F_{10} \rightarrow b_{17} ; F_{5} \rightarrow b_{18}$ & 358.8 \\
8 & $F_{6} \rightarrow b_{2} ; F_{6} \rightarrow b_{8} ; F_{9} \rightarrow b_{14} ; F_{2} \rightarrow b_{22} ; F_{1} \rightarrow b_{23}$ & 330.8 \\
9 & $F_{3} \rightarrow b_{2} ; F_{8} \rightarrow b_{6} ; F_{6} \rightarrow b_{11} ; F_{4} \rightarrow b_{12} ; F_{9} \rightarrow b_{14} ; F_{7} \rightarrow b_{19} ; F_{2} \rightarrow b_{22} ; F_{1} \rightarrow b_{23}$ & 521.6 \\
10 & $F_{3} \rightarrow b_{3} ; F_{8} \rightarrow b_{6} ; F_{5} \rightarrow b_{7} ; F_{4} \rightarrow b_{12} ; F_{10} \rightarrow b_{17} ; F_{2} \rightarrow b_{21}$ & 335.4 \\
\hline
\end{tabular}

According to the corresponding task assignment schemes and cost of warehouse robots of each order in Table 4, the total operation cost for the warehouse robots to complete all tasks within 10 a.m. and 12 a.m. is 4,079.4 dollars.

In the task assignment scheme $F_{6} \rightarrow b_{1}$ of the fifth order and the task assignment scheme $F_{4} \rightarrow b_{12}$ of the ninth order, the warehouse robots need to walk across the warehouse for handling the task, which greatly increases the operation cost. Meanwhile, task assignment schemes $F_{4} \rightarrow b_{6}, F_{4} \rightarrow b_{7}$ in the second order, task assignment schemes $F_{8} \rightarrow b_{3}, F_{8} \rightarrow b_{5}$ in the fifth order, and task assignment schemes $F_{6} \rightarrow b_{2}, F_{6} \rightarrow b_{8}$ in the eighth order all assign a warehouse robot to carry two shelves in picking one order; however, some of warehouse robots have no work. It leads to the unbalanced distribution of warehouse robots and greatly reduces the efficiency.

From task assignment schemes of robots in the 10 orders, it can be proved that using the heuristic algorithm of this paper to assign robots, the cost was reduced by $2 \%$ and it can effectively avoid far route and unbalanced workload of robots and so on. The working efficiency of warehouse robots has been improved greatly and the operation cost of warehouse robots has been reduced.

\section{Conclusions}

In the paper, the TARSWE based on cargo-to-person is investigated. The mathematical model of task assignment problem for picking one order is established to reduce the total operation cost. Further, a heuristic algorithm is designed to solve the task assignment problem for picking multiple orders. At the end, by analyzing the orders data of online bookstore A, the feasibility and validity of the model and algorithm are verified.

This paper assumes that the orders are picked one by one. When the scale of the warehouse is larger, i.e., the amount of orders is larger and the quantity of warehouse robots is enough, the orders can be batched according to their similarity (Z. Li \& W. Li, 2014b), every batch of orders can be picked simultaneously, then in this case, the heuristic algorithm in this paper can also be used for the task assignment.

\section{References}

Gao, P. (2006). Multi-robot task allocation for exploration. Journal CSUT, 13, 548-551.

Guo, Y. (2010). Auction-based multi-agent task assignment in smart logistic center (ProQuest digital dissertations). 
Hazard, C., Wurman, P., \& Andrea, R. (2006). Alphabet soup: A testbed for studying resource assignment in multi-vehicle systems. Proceedings from the $\{A A A I\}$ Workshop on Auction-Based Robot Coordination.

Kaleci, B., \& Parlaktuna, O. (2012). Market-based multi-robot task allocation using energy-based bid calculations. International Journal of Robotics and Automation, 27, 396-410.

Li, G. (2012). Task assignment of warehouse robots based on intelligence optimization algorithm (ProQuest digital dissertations).

Li, Z., \& Li, W. (2014a). Study on optimization of storage bays of smart warehouses of online bookstores. Logistics Technology, $33(12), 340-342$.

Li, Z., \& Li, W. (2014b). Study on supplier warehouse location combination problem of chain supermarkets. Logistics Technology, 33(9), 137-139.

Liu, X. (2010). Application of chaos ant colony algorithm in multi-robots system mission planning (ProQuest digital dissertations).

Trigui, S., Koubaa, A., \& Cheikhrouhou, O. (2014). A distributed market-based algorithm for the multi-robot assignment problem. Proceedings from 20143 rd International Workshop on Cooperative Robots and Sensor Networks (Robosense-2014).

Wu, Y., \& Dong, P. (2008). A general simulated annealing algorithm for solving large scale asymmetrical assignment problem. Journal of Lanzhou Jiaotong University, 27, 149-155.

Wurman, P., Andrea, R., \& Mountz, M. (2008). Coordinating hundreds of cooperative, autonomous vehicles in warehouses. AI Magazine, 29, 9-20.

Zou, S. (2013). The present and future of warehouse robot. Logistics Engineering and Management, 35, 171-172. 